\title{
Value of the Jason-1 Geodetic Phase to Study Rapid Oceanic Changes and Importance for Defining a Jason-2 Geodetic Orbit ${ }^{\mathscr{D}}$
}

\author{
G. DibARBOuRE \\ CLS, Ramonville-Saint-Agne, France \\ R. MORROW \\ LEGOS/CTOH, Toulouse, France
}

(Manuscript received 12 January 2016, in final form 1 April 2016)

\begin{abstract}
Because of the drifting nature of the ground track of Jason-1 during its geodetic mission (GM), there are 1200 overlap events where the Jason-1 GM and Jason-2 tracks align perfectly (less than their altimeter footprint radius) over thousands of kilometers. These overlap events sample homogeneously all longitudes and all time differences $(d t)$ ranging from a few minutes to 10 days or more.

When $d t$ is almost zero, the difference is characterized by altimeter noise and its modulation by waves. As $d t$ increases, the rapid ocean variability is revealed. The first statistical analysis of the 1200 events yields variance maps, spectra, autocorrelation, and space-time scales that are consistent with past observations (e.g., the 3-day phase of ERS-1). This paper highlights the value of this Jason-1 GM overlap dataset for more sophisticated studies of the rapid ocean variability. There are two major limitations: 1) the noise level of Jason-class altimeters prevents analyzing scales smaller than $80 \mathrm{~km}$ and 2) short time differences also absorb a fraction of the derivative of slower signals.

These findings are important if a geodetic mission is considered for Jason-2 in the coming years (e.g., when the satellite starts to exhibit aging problems): a well-chosen geodetic orbit for Jason-2 has the potential to collect a better distribution of overlap events with Jason-3. To that extent, thousands of orbits were screened to find the options that would provide good geodetic and mesoscale sampling and also maximize the overlap sampling of a tentative Jason-2 GM phase.
\end{abstract}

\section{Introduction}

\section{a. Context}

Jason-2 was launched in June 2008. Many years later, the altimeter and the platform are still active and performing well. Yet the mission has outlived its expected life span: the nominal mission was 3 years. To that extent, the risk of an onboard anomaly or a critical failure is slowly increasing as discussed by Dibarboure and Lambin (2015). To protect the historical TOPEX/Jason orbit for

Supplemental information related to this paper is available at the Journals Online website: http://dx.doi.org/10.1175/ JTECH-D-16-0015.s1.

Corresponding author address: Gerald Dibarboure, CLS, 8-10 Rue Hermes, 31520 Ramonville St-Agne, France.

E-mail: gerald.dibarboure@cls.fr
Jason Continuity of Service (Jason-CS)/Sentinel-6, the space agencies may decide to move Jason-2 to a so-called extension-of-life (EoL) orbit, when the risk of losing control of the satellite is judged to be too high.

This situation occurred in the past with Jason-1, and Dibarboure et al. (2012) proposed a strategy to maximize the science return of the Jason-1 EoL orbit. At that stage, Jason-2 and Jason-1 were in an interleaved tandem orbit, providing optimal space-time coverage of the mesoscale field. By moving Jason-1, the sampling capability was reduced. Moreover, moving to a new ground track involved a small increase in the error budget, due to gridded mean sea surface model errors. Conversely, the EoL phase made it possible to put Jason-1 on a drifting orbit that would accommodate the needs of the geodetic community, and various orbit options were selected in order to collect a useful geodetic dataset.

Jason-1 was moved to its new orbit in 2012 and the geodetic mission (GM) lasted for approximately 
400 days. Le Traon et al. (2015) recently reported the diminished but still substantial value of Jason-1 GM for operational oceanography, and Sandwell et al. (2014) reported the unprecedented benefits of extending the Jason-1 mission with an orbit for geodetic studies.

\section{b. Objectives}

This paper focuses on a small subset of the Jason-1 GM dataset: there are approximately 1200 so-called overlap events between the Jason-2 and Jason-1 GM. During these events, both satellites stay within $10 \mathrm{~km}$ (altimeter footprint radius) and 10 days from one another over tens of thousands of kilometers, thus providing unprecedented observations on the changes in ocean topography at time scales ranging from a few minutes to 10 days (less than a Jason-2 repeat cycle).

This series of Jason-2-Jason-1 GM overlap events shares some properties with the ERS-1 3-day repeat orbit analyzed by Minster and Gennero (1995). Both datasets provide a way to measure higher frequencies than the 10-day repeat orbit of the TOPEX/Jason orbit over thousands of kilometers. However, the difference between Jason-2 and Jason-1 benefits from better precision (their instrument noise is lower) and accuracy (the orbit determination error is an order of magnitude lower on Jason-class missions). Jason-1 GM overlap events also observe 0- to 10-day differences, whereas ERS-1 was limited to multiples of 3 days.

These new observations are interesting because rapid sea level time series are usually observed at point locations from deep ocean moorings or tide gauges and rarely over large space scales. Sea level variability can be impacted by the instrument noise and surface roughness conditions, which will be dominant at near-zero lag. Ocean dynamics at 1-10-day time scales include weak diurnal signals, internal waves and tides, the development of submesoscale fronts and filaments or small-scale eddies, the displacement of larger eddies, meanders or planetary waves, and the distinct rapid equatorial trapped baroclinic gravity waves, with spectral peaks of a few days (e.g., Farrar and Durland 2012). These rapid dynamics play an important role in ocean adjustment and mixing, but their spatial/meridional structure is difficult to observe, yet observations are required to validate their behavior in models.

This paper presents the first analyses of these overlap events in order to illustrate their value for the observation of rapid oceanic dynamics (section 2). In section 3, we use these findings to revisit the EoL strategy developed for Jason-1. Additional criteria are used to find a tentative Jason-2 EoL orbit that would not only maximize the science return for operational oceanography and geodesy but also collect welldistributed overlap events between Jason-2 and Jason-3.

\section{c. Orbit nomenclature and properties}

In this paper, we use the $N+P / Q$ orbit terminology and the notion of subcycles detailed in Dibarboure et al. (2012). The three integers $N+P / Q$ are used to define unambiguously one fractional number that corresponds to the exact number of satellite revolutions per nodal day. The fractional number $N+P / Q$ thus defines the satellite altitude and the orbit repeat cycle. ${ }^{1}$ The integers $N$ and $P$ are meaningless in this study (only the fractional number $N+P / Q$ matters), and $Q$ defines the repeat cycle in nodal days. Nodal days are defined as the time required for the earth to rotate once with respect to the orbital plane [i.e., almost but not exactly one day due to orbital precession; explained in section 8.1.4 of Chelton et al. (2000)].

Subcycles are sometimes called near-repeat cycles (as opposed to exact repeat cycles). Dibarboure et al. (2012) explain how the so-called subcycles of geodetic orbits create an apparent "zonal drift" with respect to the fixed grid of Jason-2.

\section{d. Data used}

In this paper, we use level 3 along-track sea level anomalies (SLA) from AVISO (2015), described by Dibarboure et al. (2011). More specifically we use the "calibrated SLA" product of the AVISO catalogue [explained and analyzed in section $2 \mathrm{~g}(1)$ ]. AVISO (2015) gives the list of corrections and references they used to construct the sea level anomalies. Jason-2 SLA are computed as the difference between the sea surface height (SSH) and a local mean sea surface (also known as mean profile) based on 20 years of SSH from TOPEX/ Poseidon to Jason-2. During its geodetic phase, the SLA from the Jason-1GM is calculated relative to a gridded mean sea surface model instead (CNES/CLS, version 2011). This difference is analyzed and discussed in section $2 \mathrm{~g}(3)$.

\section{Using Jason-1 GM to study rapid oceanic changes}

\section{a. Collocating Jason-1 GM and Jason-2 to build new observations}

An important feature of the Jason-1 GM orbit is that it has the same inclination and almost the same altitude as

\footnotetext{
${ }^{1}$ Jason-2 performs 12.7 (i.e., $12+7 / 10$ ) revolutions per nodal day, which results in an exact repeat cycle of 10 nodal days or 9.91 calendar days after 127 revolutions. In contrast, the Jason-1 GM orbit performs $12+299 / 410$ (i.e., approximately 12.729 ) revolutions per nodal day, which results in a very long exact repeat cycle of 410 nodal days (approximately 406 calendar days).
} 

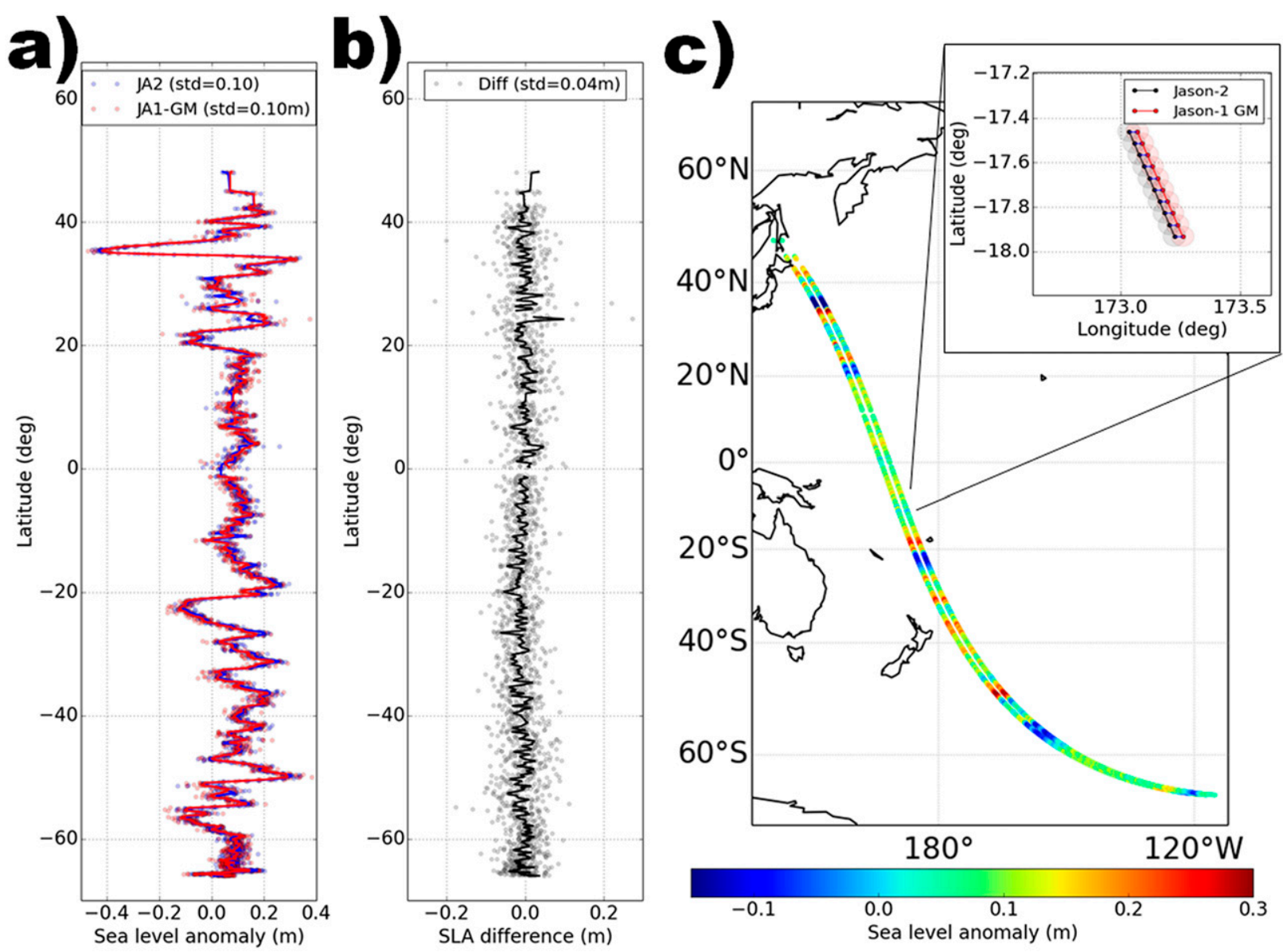

FIG. 1. Comparison of Jason-2 and Jason-1 GM when both satellites are separated by less than 20 min and flying over the same ground track (less than $5 \mathrm{~km}$ from one another). (a) The along-track SLA of each altimeter (Jason-2 in black, Jason-1 GM in red). (b) The difference between both datasets (raw data in gray, smoothed profile in black). (c) The position of both tracks (Jason-1 GM is shifted by $2^{\circ}$ to avoid a perfect overlap with Jason-2) with a zoom highlighting the altimeter footprint of each altimeter.

Jason-2. The consequence is that both datasets have a ground track with the same geometry: the tracks of Jason-2 and Jason-1 GM sometimes align perfectly over an entire orbit revolution or more. Note that they can be measuring topography at the same position (spatial offset is less than $10 \mathrm{~km}$ ) either at the exact same time or not (temporal offset ranges from 0 to 10 days).

In section $2 b$, we illustrate this property by selecting three samples out of the 1200 overlap events where the Jason-1 GM and Jason-2 tracks are within $10 \mathrm{~km}$ and 10 days from one another. The space and time distribution of the 1200 overlap events is then analyzed in section 2c. Last, a statistical analysis of all overlap events is carried out in sections $2 \mathrm{~d}-\mathrm{f}$.

\section{b. Sample overlap events from 20 min to 10 days}

To illustrate the overlap methodology, we first select an overlap event occurring in the Pacific Ocean (Fig. 1) where satellites are measuring the topography within
$5 \mathrm{~km}$ and $20 \mathrm{~min}$ from one another. As expected, Fig. 1a shows that the ocean topography measured by both sensors is essentially the same: it ranges from -40 to $30 \mathrm{~cm}$ with a standard deviation of $10 \mathrm{~cm}$. Figure 1c illustrates that the geometry of both tracks is identical (Jason-1 GM is shifted by $2^{\circ}$ in order to be visible on the map) and that both SLA profiles are almost identical. The thumbnail zoom in Fig. 1c also gives an overview of how the altimeter footprints (here approximated as 10-km-radius circles) overlap.

Figure $1 \mathrm{~b}$ shows the difference between both SLA profiles. This residual has a standard deviation of $4 \mathrm{~cm}$. This value should be compared to the intrinsic $1-\mathrm{Hz}$ noise level of Jason-class altimeters: globally on the order of $2.7 \mathrm{~cm}$ at $1 \mathrm{~Hz}$ and modulated by significant wave height. Dibarboure et al. (2014) have reported that the $1-\mathrm{Hz}$ noise for Jason-1 and Jason-2 are not correlated, so one would expect the differences of Fig. 1b to yield a standard deviation of $\operatorname{sqrt}(2) \times 2.7 \mathrm{~cm}=3.8 \mathrm{~cm}$, that is, very close 

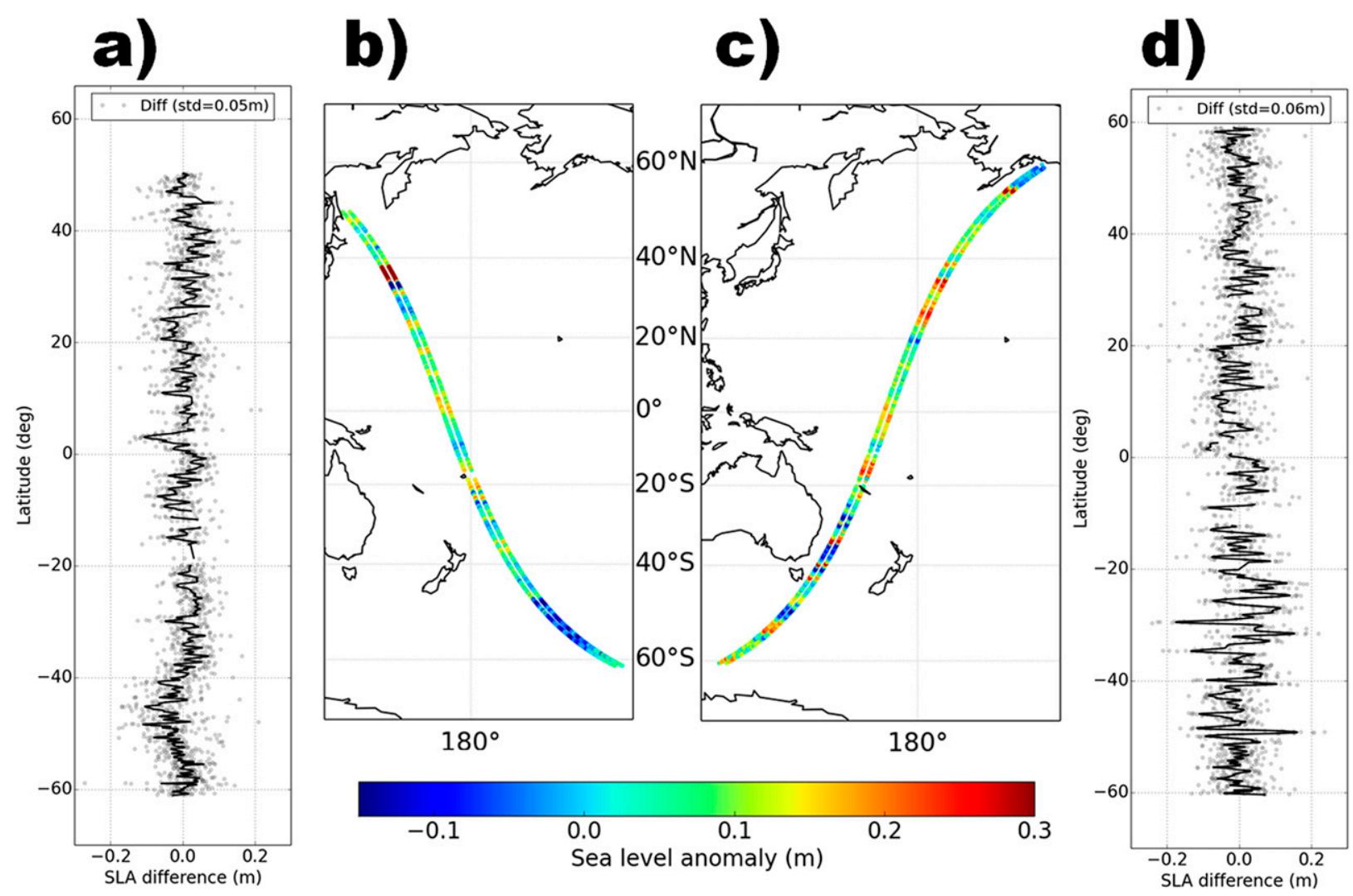

FIG. 2. As in Figs. 1b and 1c, but for Jason-2 and Jason-1 GM samples separated by (left) 2 and (right) 5 days.

to what is found in this sample. There is approximately sqrt $\left(4^{2}-3.8^{2}\right)=1.2 \mathrm{~cm}$ RMS missing, which could be explained by the wave modulation of noise on this specific sample or by an additional mean sea surface (MSS) error on Jason-1 GM that is discussed by Dibarboure et al. (2012) and analyzed in section $2 \mathrm{~g}(1)$. Figure $1 \mathrm{~b}$ also shows that the difference is mainly composed of high wavenumbers, as expected from a difference dominated by such errors.

The noise floor of Jason-1 should be compared to the 4-4.5 $\mathrm{cm}$ reported by Minster and Gennero (1995) for the 3-day orbit of ERS-1. To smooth out the influence of instrumental noise of ERS, they generated sea level anomalies with a 10-km resolution using a Lanczos filter with a $60-\mathrm{km}$ cutoff wavelength, whereas we use standard $1-\mathrm{Hz}(7 \mathrm{~km})$ products and apply no smoothing whatsoever.

We also selected a few overlap events with a longer time difference to illustrate the contribution from rapid ocean changes and, in particular, from rapid mesoscale dynamics. Figure 2 shows the difference in sea level anomaly for two Jason-1-Jason-2 overlaps with a 2- and 5 -day lag. As in the previous example, the tracks of both satellites are aligned over an entire pass in the western Pacific Ocean (Jason-1 GM stays within $10 \mathrm{~km}$ of Jason-2).

Figure $2 \mathrm{a}$ and $2 \mathrm{~b}$ (2-day lag) show that high-frequency noise is still a major component of the difference. Longer wavelengths (e.g., 100-500 km or more) that were barely visible in the 20-min overlap now become clearly visible. Similarly, the standard deviation is now $5 \mathrm{~cm}$ (vs $4 \mathrm{~cm}$ for the 20-min overlap): there is an additional $\sqrt{ }\left(5^{2}-4^{2}\right)=$ $3 \mathrm{~cm}$ RMS of additional energy in this 2-day overlap sample. The 5-day differences (Figs. 2c and 2d) have a standard deviation of $6 \mathrm{~cm}$ (i.e., $3.3-\mathrm{cm}$ RMS more than in the case of the 2-day overlap of Fig. 2a) and include a wide range of wavelengths.

\section{c. Distribution of overlap events}

There are on average three overlap events per day somewhere in the world. Figure 3 a shows the longitude of the equator crossing for some ascending passes of Jason-2 and Jason-1 GM during the first 40 days of the GM phase. The vertically aligned red dots show the 10-day repeat orbit used by Jason-2. The diagonally aligned blue dots of Jason-1 GM show the longitude drift associated with the geodetic phase. In this plot, red and blue dots are sometimes overlapping (e.g., $20^{\circ} \mathrm{E}$ and 9 days, 


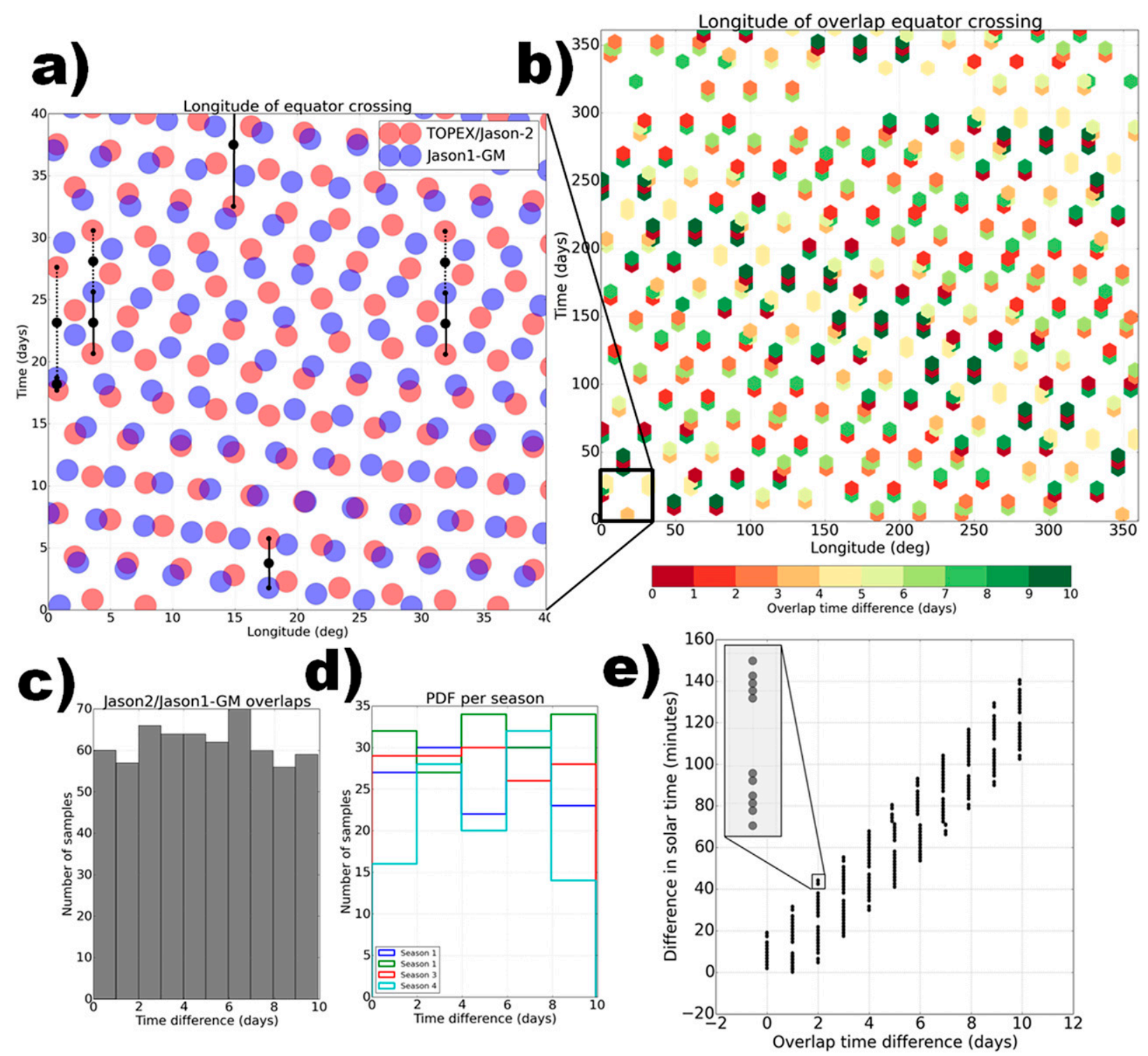

FIG. 3. Overview of the space-time distribution of overlaps between Jason-2 and Jason-1 GM (limited to ascending passes). (a) The longitude of the equator crossing of Jason-2 (red) and Jason-1 GM (blue) throughout the 40 first days of the Jason-1 GM phase. Each overlap event is highlighted with black dots and lines linking the overlapping tracks of each altimeter. (b) The longitude of the equator crossing of all events where Jason-1 GM is within $10 \mathrm{~km}$ and 10 days of Jason-2 [black lines in (a)]. (c) The distribution of the time difference between Jason-1 and Jason-2 measurements during these overlap events. (d) As in (c), but split into four subsequent periods of 90 days. (e) The difference in solar time between Jason-1 GM and Jason-2, as a function of the overlap time difference.

respectively): these are overlap events where the spatial distance is small and the temporal distance is less than a few hours. More frequently, however, the blue dots are vertically aligned with the red dots: these are overlap events for which the temporal offset ranges from 1 to 10 days. These events are highlighted with black lines and dots linking the Jason-2 and Jason-1 GM tracks.

A search algorithm can locate all of the overlap events (Fig. 3b, only ascending passes are shown for the sake of clarity). Each dot represents an entire ascending arc from $66^{\circ} \mathrm{S}$ to $66^{\circ} \mathrm{N}$; Fig. $3 \mathrm{~b}$ shows their distribution throughout the entire GM phase. Note that many dots are missing from days 290 to 350 due to missing data on either satellite.

The color of each dot in Fig. $3 b$ gives the time difference between both datasets. The overlaps often come in couples: if Jason GM is over a Jason- 2 track with a $\delta \mathrm{t}$ time difference, then it will also provide an overlap with the previous or next Jason-2 cycle ( +10 days or -10 days), which results in a second overlap at $10-\delta t$. The overlap 
events also occur in clusters. It is not possible to find overlap events in all regions at all times, especially if one is looking for a specific delta time (e.g., to study the oceanic variability faster than 3 days). Fortunately, the Jason-1 GM orbit yields a reasonably homogeneous overlap distribution illustrated by Fig. 3c, where the PDF of overlap time differences is nearly constant, with an average of 60 samples per 1day time bin for $0-10$-day lags.

This homogeneous distribution allows us to carry out statistical studies on the 1200 available samples. To illustrate, the estimation error of the mean SSH spectrum is governed by chi-squared statistics, having twice as many degrees of freedom as the number of samples. So, with 120 independent samples per time difference (e.g., for a 3-day lag), the upper bound of the mean 3-day spectrum uncertainty is on the order of $15 \%$. This is enough to derive a global statistical analysis of the 1200 overlap events given by Jason-1. This dataset might be biased by specific intense events or data gaps near the end of the GM phase; however, because of their homogeneous distribution in space and over 400 days, the global results are unlikely to be skewed by an irregular temporal or spatial aggregation (e.g., as opposed to the 3-month dataset collected during the 3-day phase of ERS-1).

Yet the number of samples collected with Jason-1 GM is still limited for regional or seasonal analyses: with approximately 15-30 samples per season (Fig. 3d), the uncertainty of a mean spectrum would be as high as a $30 \%-50 \%$.

Last, Fig. 3e shows that the solar time of Jason-1 GM barely drifts away from the solar time of Jason-2, essentially because their altitudes are almost the same. At the beginning of the GM phase, the offset in solar time seen with 0 -day differences is $\sim 5 \mathrm{~min}$; and after 400 days of the GM phase, they are separated by $20 \mathrm{~min}$. Furthermore, the solar time of both Jason satellites changes by approximately $12 \mathrm{~min}_{\text {day }}{ }^{-1}$ (due to a 120 -day period of the so-called orbit beta-prime angle), so all overlap events with a time difference of $D$ days will exhibit a solar time difference of $12 D \mathrm{~min} \pm 20 \mathrm{~min}$. In other words, the time difference in days is almost an integer value: it is possible to find solar time differences ranging from -2 to $2 \mathrm{~h}$, but it is not possible to find overlaps with a solar time offset ranging from 3 to $21 \mathrm{~h}$. This is a limiting factor to study tides and diurnal effects, since diurnal harmonics largely cancel out in the Jason-2-Jason-1 difference unless they are rapid nonstationary events.

\section{d. Spatial properties}

We have collocated the Jason-1 GM and Jason-2 tracks during overlap events. We split each sample into subsets of approximately $600 \mathrm{~km}$, avoiding coastal zones
(50 km from the coast) and large data gaps (gaps of $20 \mathrm{~km}$ or less were filled by linear interpolation). Overlap events were also divided into bins of time differences $(d t)$ ranging from 0 to 60 days. For visual representation, 1-day bins are shown from 0 to 10 days and 5-day bins are used from 10 to 60 days (e.g., the 58-day bin includes time differences ranging from 56 to 60 days).

Figure 4 shows the global statistical analysis of the overlap events. Figure $4 \mathrm{a}$ shows that the SLA difference has a very clean bell-shaped distribution, very peaky for 0 -day differences (as expected from $1-\mathrm{Hz}$ noise) and widening as the time difference increases. The associated standard deviations range from $4 \mathrm{~cm}$ for 0 -day differences to $7 \mathrm{~cm}$ for 9-day differences. The samples from section $2 \mathrm{~b}$ are representative of this global distribution.

If the signals measured by Jason-1 and Jason-2 are uncorrelated, then the variance of their difference will measure the sum of both sample variances. To illustrate, a 60-day difference in Fig. 4a would exhibit a very wide bell-shaped curve with a standard deviation larger than the Jason-2 standard deviation by a factor of sqrt(2). To that extent, the power spectra from Fig. $4 \mathrm{~b}$ were normalized by a factor of 0.5 in order to be easily comparable with the black spectrum of Jason-2. Furthermore, the smaller scales from 15 to $30 \mathrm{~km}$ cannot really be trusted as collocation/reinterpolation behaves like a low-pass filter. We have highlighted this limit as a gray box on the power spectral density (PSD).

At 0 day, the spectrum is almost flat and is dominated by instrument noise, as expected from the observations of section $2 \mathrm{~b}$. However, the 0-day spectrum is not perfectly flat. This contrasts with $20-\mathrm{Hz}$ observations from Dibarboure et al. (2014) during the Jason-2Jason-1 tandem phase, where both altimeters yield a perfectly flat spectrum when they were observing exactly the same surface within $1 \mathrm{~min}$ and $300 \mathrm{~m}$ from one another. In overlap events, Jason- 1 can be as far as $10 \mathrm{~km}$ and $20 \mathrm{~min}$ away from Jason-2, resulting in small changes in wind/wave or MSS effects that are not be perfectly accounted for in current altimetry products [discussed in section $2 \mathrm{~g}(3)$ ].

More importantly, the 1-day (purple) to 5-day (cyan) spectra of Fig. $4 \mathrm{~b}$ show a rapid increase of the PSD for wavelengths $\lambda>60-70 \mathrm{~km}$. To illustrate, near $\lambda \sim 300 \mathrm{~km}$ the PSD of a 1-day difference exhibits 2.5 times more energy than 0-day differences, and the energy is doubled again from 1- to 2-day differences. Furthermore, 5-day difference spectra have as much energy as $10 \%$ of the PSD of Jason-2. For longer time differences, the difference spectra increase asymptotically toward the spectrum of Jason-2.

This finding is surprising for two reasons: 1 ) the variance increase is faster than that reported by Minster and 

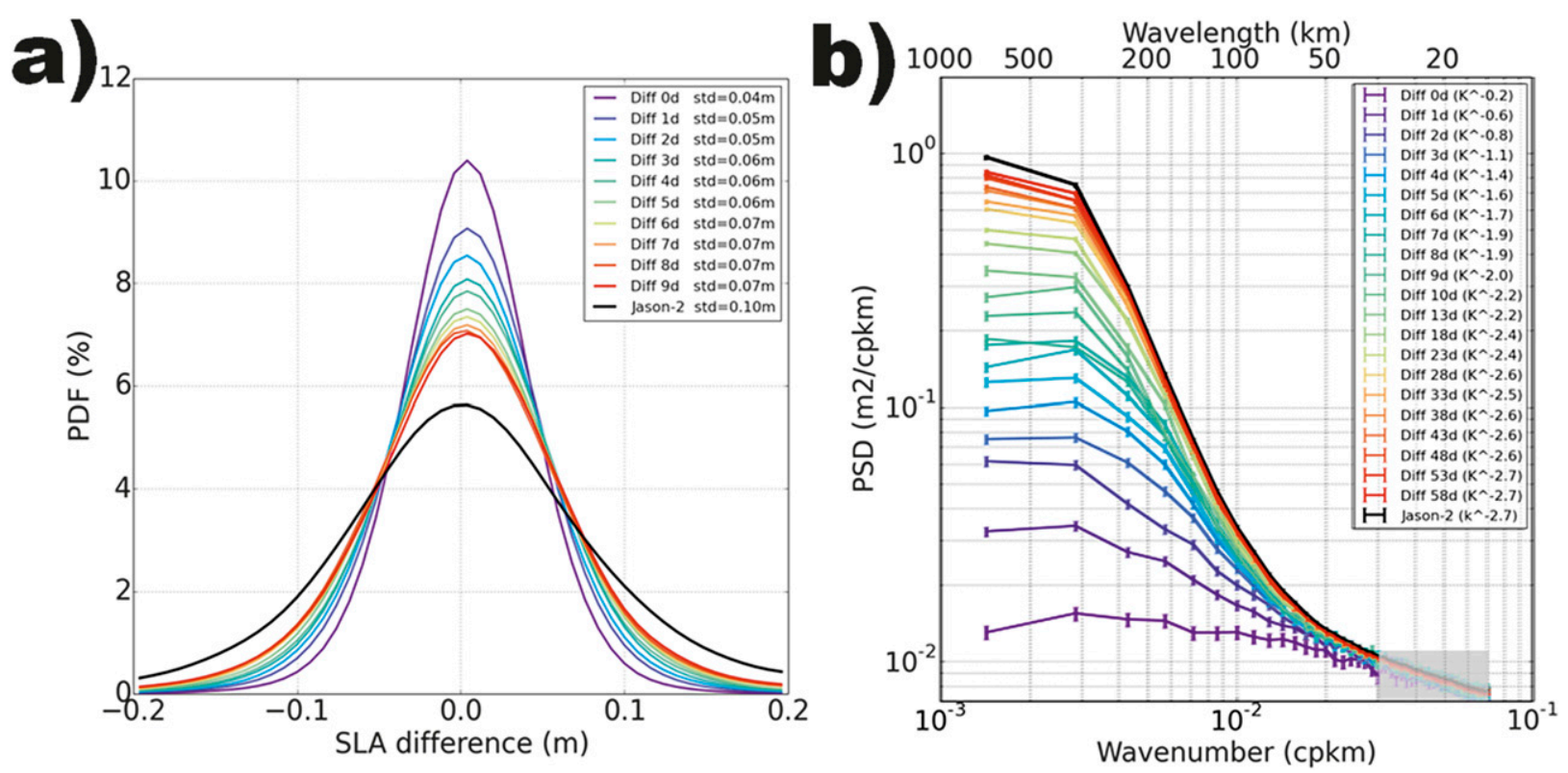

FIG. 4. Global statistical analysis of the Jason-1 GM-Jason-2 overlap events. (a) The PDF of the difference from 0- to 9-day differences (purple to red). (b) The mean global spectrum of Jason-2-Jason-1 GM for 0- to 60-day (purple to red) overlaps. Jason-2 reference curves are in black.

Generro (1995) with ERS-1, or by Le Traon (1991) with Geosat data; and 2) the PSD primarily increases for wavelengths that are relatively long, rather than the smaller, rapidly moving signals.

This could be explained by two reasons.

1) First, the differences between Jason-1 and Jason-2 are limited by the noise floor (i.e., 0-day spectrum): it is not possible to observe any signal smaller than the observability limit of a 4-cm white noise (Dufau et al. 2016). The noise floor of a Jason-class mission is approximately 2.5 times lower than for $E R S$-1, but the noise floor is doubled in the Jason-2 to Jason-1 differences. Consequently, we are primarily inferring rapid changes in the larger scales that are more energetic than twice the noise floor.

2) Second, a 1- or 3-day difference is not a perfect measurement of the 1- or 3-day variability: the difference is also capturing a fraction of the derivative of slower signals (discussed in the appendix). For instance, assuming that a given temporal PSD is following an $f^{-2}$ law as reported by Wortham and Wunsch 2014), time differences are merging a wide range of low frequencies (also true for $f^{-1}$ or $f^{-3}$ ). This is also a limit of the 1D profiling nature of nadir altimeters: even for slow and stable eddies such those analyzed by Samelson et al. (2014), our overlap events are only isolated profiles across a small subset of eddies or meanders.

\section{e. Correlation and coherence}

Figure 5a shows the autocorrelation function for 0- to 60-day differences. The autocorrelation of Jason-2 is also given. The 0-day curve has a Dirac delta shape expected from a difference dominated by white noise. In contrast, 1 day and beyond exhibit typical SLA autocorrelations (e.g., Le Traon et al. 1990) with a small negative lobe and 0 -crossing values ranging from $60 \mathrm{~km}$ for 1-day differences to slightly more than $100 \mathrm{~km}$ for 60-day differences and Jason-2. Our curve for the global ocean is consistent with regional values reported by Stammer (1997) on the order of $120 \mathrm{~km}$ in the tropical band and $60-80-\mathrm{km}$ scales for mid- to high latitudes. One might intuitively expect even shorter zero-crossing values for 1- to 5-day time differences, but this is likely explained by reasons 1 and 2 above.

Similarly, Fig. 5b shows the coherence $\gamma^{2}$ between Jason-1 GM and Jason-2 as colored 1D curves for 0- to 60-day lags, which exhibit a sigmoid shape. For 0-day differences, the coherence is almost 1 for long wavelengths and it decreases for higher wavenumbers as the incoherent noise of the altimeters becomes dominant.

Assuming that both Jason satellites are measuring exactly the same ocean spectrum $S(1 / \lambda)$ for wavelength $\lambda$ and an independent noise $\mathcal{E}_{i}$, we can define $\alpha(1 / \lambda)$ as the ratio between the PSD of the noise floor and the PSD of the signal. In this simple case, the coherence can be approximated by Eq. (1). The key value of $\gamma^{2}=0.25$ is reached when $\alpha=1$, that is, when the PSD of the 

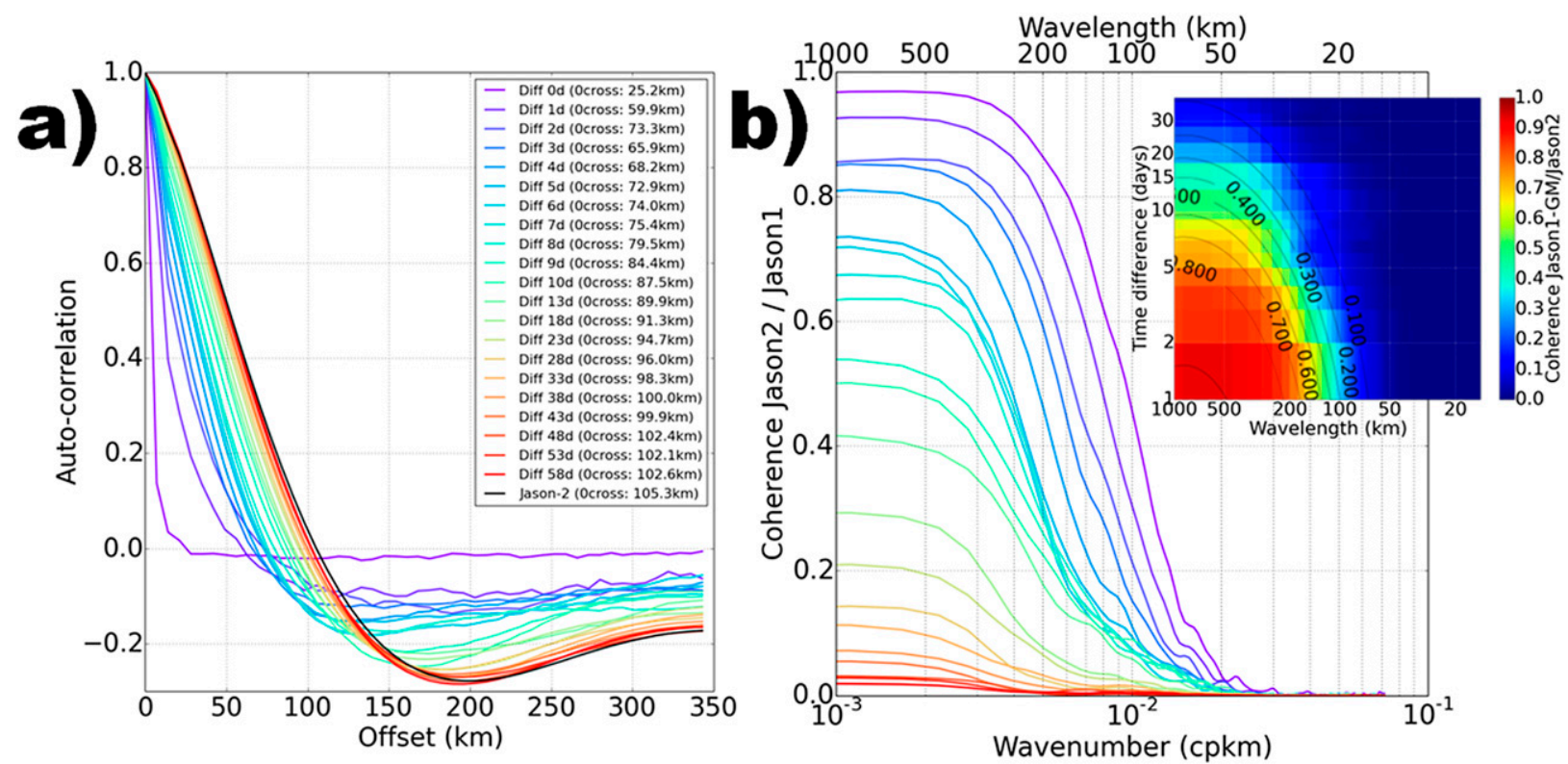

FIG. 5. Global statistical analysis of the Jason-1 GM-Jason-2 overlap events. (a) The mean global autocorrelation with zero-crossing values. (b) The $\gamma^{2}$ between Jason-1 GM and Jason-2 for 0- to 60-day (purple to red) overlaps. The inset in (b) is the same coherence shown in $2 \mathrm{D}$ (colored background) superimposed with the analytical model of Eq. (2) (black lines).

ocean signal reaches the noise floor. This defines the observability limit of ocean dynamics with a signal-tonoise ratio larger than one (Dufau et al. 2016). For our global coherence of 0-day overlap events, this point is reached for $\lambda \sim 81 \mathrm{~km}$ : on average, the noise of Jasonclass altimeters prevents us from analyzing scales less that $80 \mathrm{~km}$ in Jason-2-Jason-1 differences:

$$
\gamma^{2}(1 / \lambda)=\frac{1}{[1+\alpha(1 / \lambda)]^{2}}
$$

The coherence also decreases as the time difference increases: other colored curves are still sigmoid shaped, but their upper plateau is reached at 0.9 for a 1-day lag, 0.8 for a 4-day lag, etc. The coherence is still affected by the presence of white noise, but here the alpha parameter also contains the rapid ocean variability that decorrelates between Jason-1 and Jason-2.

The insert in Fig. 5b shows the coherence as a 2D image where each grid line is one of the colored $1 \mathrm{D}$ curves. The thumbnail is shown in logarithmic scale and as a function of the wavelength $\lambda$ and time difference $d t$. In addition to the coherence loss from the instrument noise (right-hand side limit near $80 \mathrm{~km}$ ), an ellipsoidal feature is also visible: this figure gives an order of magnitude of how fast the shorter ocean signals decorrelate with time. It can be approximated with the 2D sigmoid-type model below:

$$
1-1 /\left(1+e^{-8\left(\sqrt{(\log \lambda+3)^{2}+0.4[\log (d t)+0.5]^{2}}-1\right)}\right) .
$$

Of interest in Eq. (2) is the square root, which gives some insight about the spatial and temporal decorrelation. For example, the coherence level of 0.25 (blue/cyan) is reached for $d t=21$ days when $\lambda>300 \mathrm{~km}$ and only $d t=10$ days when $\lambda \sim 150 \mathrm{~km}$. This is consistent with the values reported by Le Traon (1991), where $\gamma^{2} \sim 0.1$ for $d t=17$ days and $\lambda \sim 200 \mathrm{~km}$. Therefore, our 2D coherence plot complements the existing models obtained with exact repeats ranging from 10 days (TOPEX/Poseidon) to 17 days (Geosat).

\section{f. Geographical distribution and temporal properties}

The regional variability can be inferred by binning the RMS of 0 - to 9-day differences: the maps from Fig. 6a show the result in $10^{\circ} \times 10^{\circ}$ boxes. The map of 0 -day differences is very homogeneous, although the RMS increases in zones of higher significant wave height values (which modulates the altimeter noise). If we limit the analysis to the high wavenumbers by high-pass filtering the SLA with a 60-km cutoff on Jason-1 and Jason-2, then the geographical distribution is similar for all maps from 0- to 9-day lags (not shown): for these wavelengths, the distribution is not affected by the temporal offset because the instrument noise dominates.

In contrast, as soon as the time difference is not zero, various regions start to emerge in Fig. 6a. With 1-day time differences, an increase in RMS is visible in the western boundary currents and the Antarctic circumpolar region. Rapid mesoscale changes or offsets in sampling of less than $10 \mathrm{~km}$ are clearly dominating the 

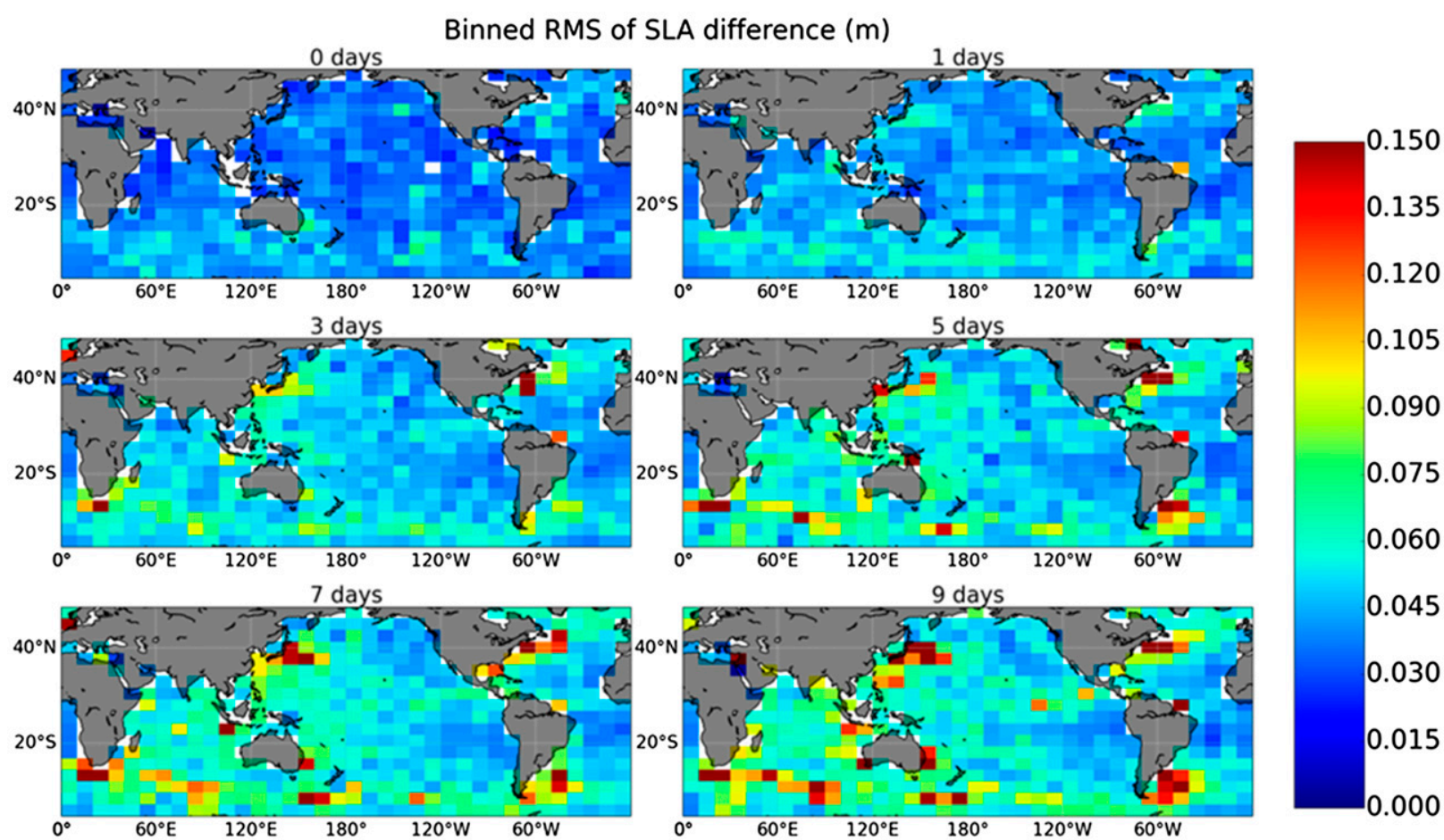

0.045
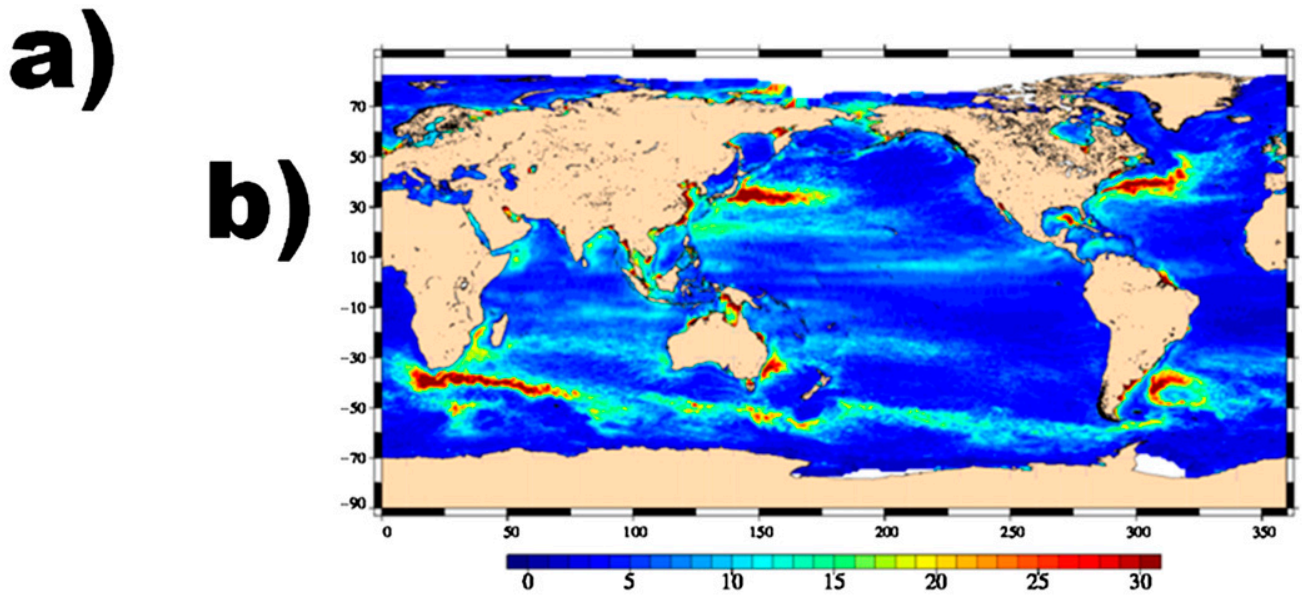

FIG. 6. (a) The binned RMS of Jason-1 GM-Jason-2 differences during overlap events ranging from 0 days (upper left) to 9 days (lower right). (b) The 1993-2012 mesoscale variability derived from AVISO maps.

"noise" distribution evident at zero lag. By 3-5 days, energy is also apparent in the western tropical Pacific, the eastern Indian Ocean, and in the intertropical convergence zones.

Figure 7 provides an overview of the variance increase for a small set of regions: the Gulf Stream, Kuroshio, northeast Pacific, tropical Pacific, and Mediterranean Sea. Figure 7a shows a linear increase of the variance in most regions from 0 to 10 days, and Fig. $7 \mathrm{~b}$ shows that the variance of the difference becomes asymptotically flat as $d t$ increases beyond 10 days. This happens rapidly in the lower energy regions and after 30-40 days in the western boundary currents. To measure the associated $e$-folding time, we use the simple analytical model given by Eq. (3). Table 1 also shows that the $e$-folding radius varies from 19 to 42 days in the different regions and is 26 days for the global oceans (Fig. 7c). The global value is longer than that obtained by Minster and Gennero (1995) with ERS-1 (e-folding time less than 20 days in strong currents), but it is consistent with the values found by Stammer (1997) with TOPEX/Poseidon or Le Traon (1991) with Geosat:

$$
V(d t)=K\left(1-e^{-d t / \tau}\right) .
$$



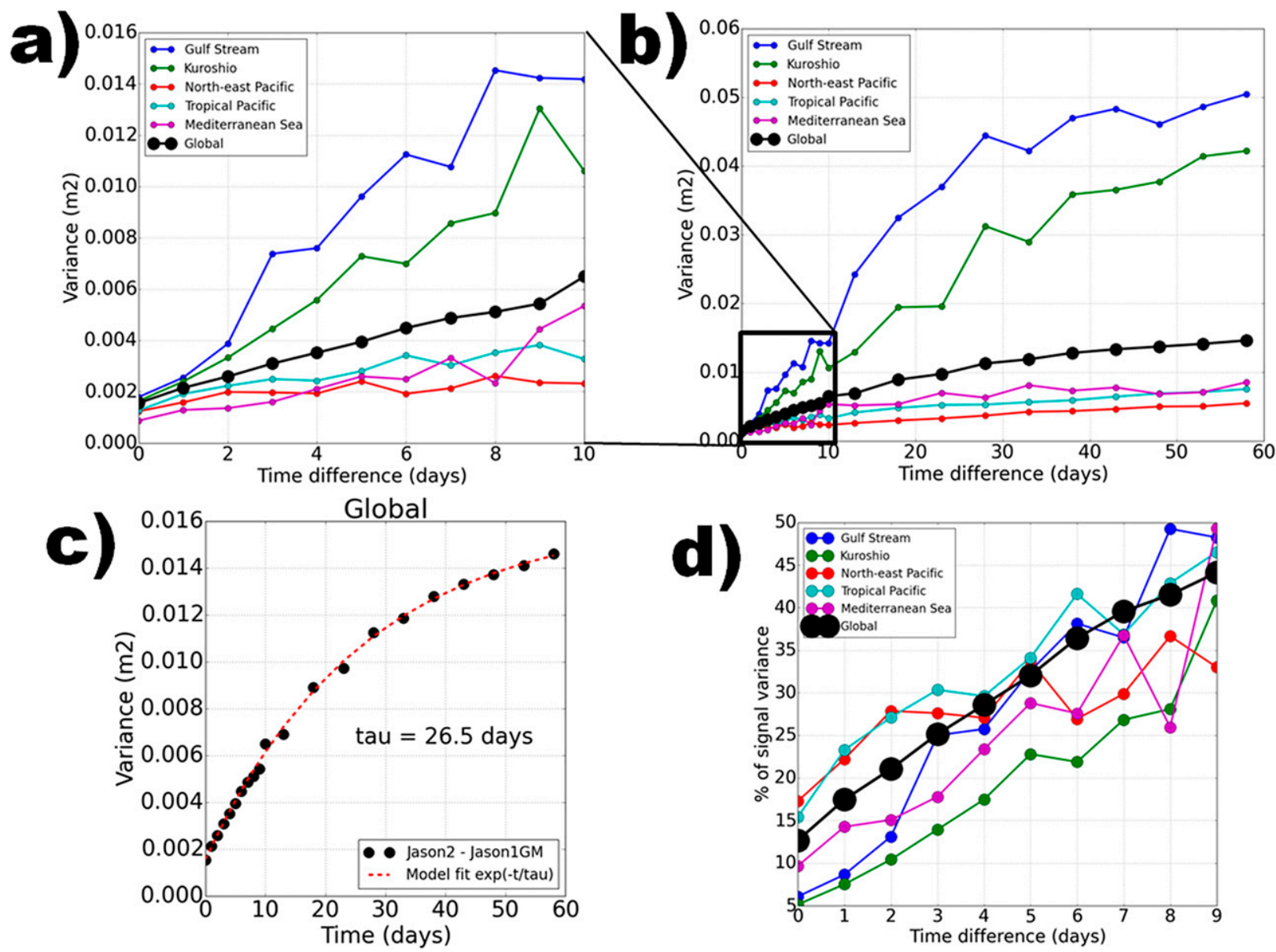

FIG. 7. Evolution of the global (black) and regional (colors) variance of the Jason-2-Jason-1GM difference as a function of the $d t$ between both samples. (a) The result for 0- to 10-day differences and (b) 0- to 60-day differences. (c) The result of an analytical model fit. (d) As in (a), but expressed in percentage of the signal variance measured by Jason-2.

The difference with Minster and Gennero (1995) is probably explained by the nature of the observations. They obtained their temporal radiuses using a $2 \mathrm{D}$ spectral analysis in space and time (repeat orbit), whereas we measure the variance in time differences. As a result, our observation also captures a fraction of the variance of slower dynamics (see section $2 \mathrm{~d}$ ), which results in measured $e$-folding radiuses that are longer than what would be observed with a true repeat cycle of one day.

This effect is also visible in Fig. 7d, which is the same as Fig. $7 \mathrm{a}$ but given in percentage of the signal variance measured by Jason-2. The 0-day differences show that the measurement noise accounts for $5 \%-15 \%$ of the total variance (note that white noise is often filtered out by the authors cited above), and the variance increases to $30 \%-$ $50 \%$ of the Jason-2 signal variance for 9-day lags. This is higher than that observed by Minster and Gennero (1995) with their 2D spectral analysis. To that extent, these results emphasize the difficulty of disentangling the variance of the rapid variability from the modulation of the slow mesoscale that is captured in these observations (the appendix).

\section{g. Discussion}

\section{1) INFLUENCE OF THE INPUT DATASETS}

We have carried out this study with a so-called calibrated level 3 along-track sea level anomaly product

TABLE 1. The $e$-folding radius obtained with a fit of the analytical model on the curves from Fig. 7b. Figure 7d illustrates the model fit for the global ocean.

\begin{tabular}{lc}
\hline \multicolumn{1}{c}{ Region } & $\tau$ (days) \\
\hline Global & 26.5 \\
Gulf Stream & 18.8 \\
Kuroshio & 21.2 \\
Northeast Pacific & 42.6 \\
Tropical Pacific & 34.6 \\
Mediterranean Sea & 21.5 \\
\hline
\end{tabular}


from AVISO (2015). In addition to the recently improved corrections, these calibrated data include specific steps for the long-wavelength error reduction. Dibarboure et al. (2011) describes the two-step calibration, derived from Le Traon and Ogor (1998), for the crossover minimization using Jason-2 as a reference mission, and from Ducet et al. (2000), for the multialtimeter calibration based on optimal interpolation. This contrasts with the processing used for ERS-1, TOPEX, and Geosat in the studies mentioned above.

The orbit determination of a Jason-class mission is almost an order of magnitude better than the accuracy of ERS or Geosat. Various geophysical corrections are substantially improved in current altimetry standards (e.g., better tidal models or improved dynamical atmospheric corrections). All of these parameters might affect the longer wavelengths of the Jason-2-Jason-1 differences. Furthermore, the Jason-2-Jason-1 multimission calibration mechanism described by Dibarboure et al. (2011) is less aggressive than the polynomial adjustments used in the previous studies.

We performed sensitivity studies to verify that these calibration algorithms improved the quality of all results and that they did not substantially affect our findings.

\section{2) NOISE AS A LIMITING FACTOR}

By far the most limiting factor in this type of study is the noise level of Jason-class measurements. The amplitude of $1-\mathrm{Hz}$ measurement noise is larger than the ocean signal for wavelengths smaller than $80 \mathrm{~km}$, and the global spectra at 0 -day difference shows an impact in the $80-250-\mathrm{km}$ range as well. This is amplified because the noise of both altimeters is random and uncorrelated, so it becomes additive when taking the difference between two topography profiles. Thus, our analysis of overlap events is limited by twice the Jason noise level.

Fortunately, recent studies such as Dibarboure et al. (2014), Garcia et al. (2014), and Amarouche et al. (2014) show that alternative processing techniques could be developed and applied to Jason data in order to reduce the $1-\mathrm{Hz}$ noise. In the future, these techniques would be valuable additions for the study of small-scale ocean topography, and especially for Jason overlaps because of the factor of 2 in SLA differences.

\section{3) AdDitional ERRORS IN THE GEODETIC PHASE OF JASON-1}

The 10-km limit we used for collocation between Jason-2 and Jason-1 is consistent with the radius of their altimeter footprints, yet the geoid can change a lot over a few kilometers (e.g., Dorandeu et al. 2003). Therefore, it does not cancel out in a Jason-2-Jason-1 difference and it must be removed from the SSH measurements before the comparison. Jason-1 GM is on a drifting orbit, so AVISO (2015) uses a gridded MSS, namely, the CNES/CLS, version 2011, model. This MSS model is not perfect, and it can generate geoid-related residual errors in our overlap differences.

We observed (not shown) that the variance of 0-day differences always increases when Jason-1 is shifted spatially from the true collocation with Jason-2. We observe a variance increase on the order of $6-7 \mathrm{~mm}$ RMS per kilometer. Even at distances less than $10 \mathrm{~km}$ away from the Jason-2 track, using a gridded mean sea surface models yields an additional $1-2 \mathrm{~cm}$ in RMS error, consistent with the observations from Dibarboure et al. (2012).

This MSS error remains relatively small with respect to the mean global oceanic variability measured by Jason in Fig. 4 and with respect to the noise floor (doubled in Jason-2-Jason-2 differences), which is on the order of $4 \mathrm{~cm}$ RMS. Indeed, the instrumental noise is up to 16 times greater than the MSS error in terms of spectral energy.

\section{Consequences for Jason-2}

\section{a. Link between overlap events and the orbit to be used for Jason-2 EoL phase}

These Jason-1 GM results could be important for Jason-2. If there is a serious risk of losing control of the satellite, then Jason-2 will be moved to a different orbit (EoL orbit) following the precedent established for Jason-1. Moreover, because Jason-1 was lost on its geodetic orbit, it is likely that Jason-2 will have to use a different GM orbit to reduce the risk of collision with the now uncontrolled Jason-1 platform.

Dibarboure et al. (2012) defined a strategy and a handful of candidates for the Jason-1 EoL orbit, but they did not anticipate the notion of overlap events. Consequently, the options they proposed are not optimal with respect to the potential to use Jason-2 EoL and Jason-3 to study rapid mesoscale changes.

To illustrate this point, Fig. 8 shows an overview of the overlap events between Jason-3 (assumed to be on the historical TOPEX track) and a worst-case Jason-2 EoL orbit $(12+286 / 399$, selected arbitrarily). Instead of a well-distributed series of overlap couples, this EoL orbit would aggregate all the overlaps in very dense clusters: over a period of 90 days, all the equator crossings of overlap events would be located in a common $180^{\circ}$ longitude band, leaving the rest of the ocean unobserved; and each cycle would sample a different zone, making such a dataset more difficult to interpret. 


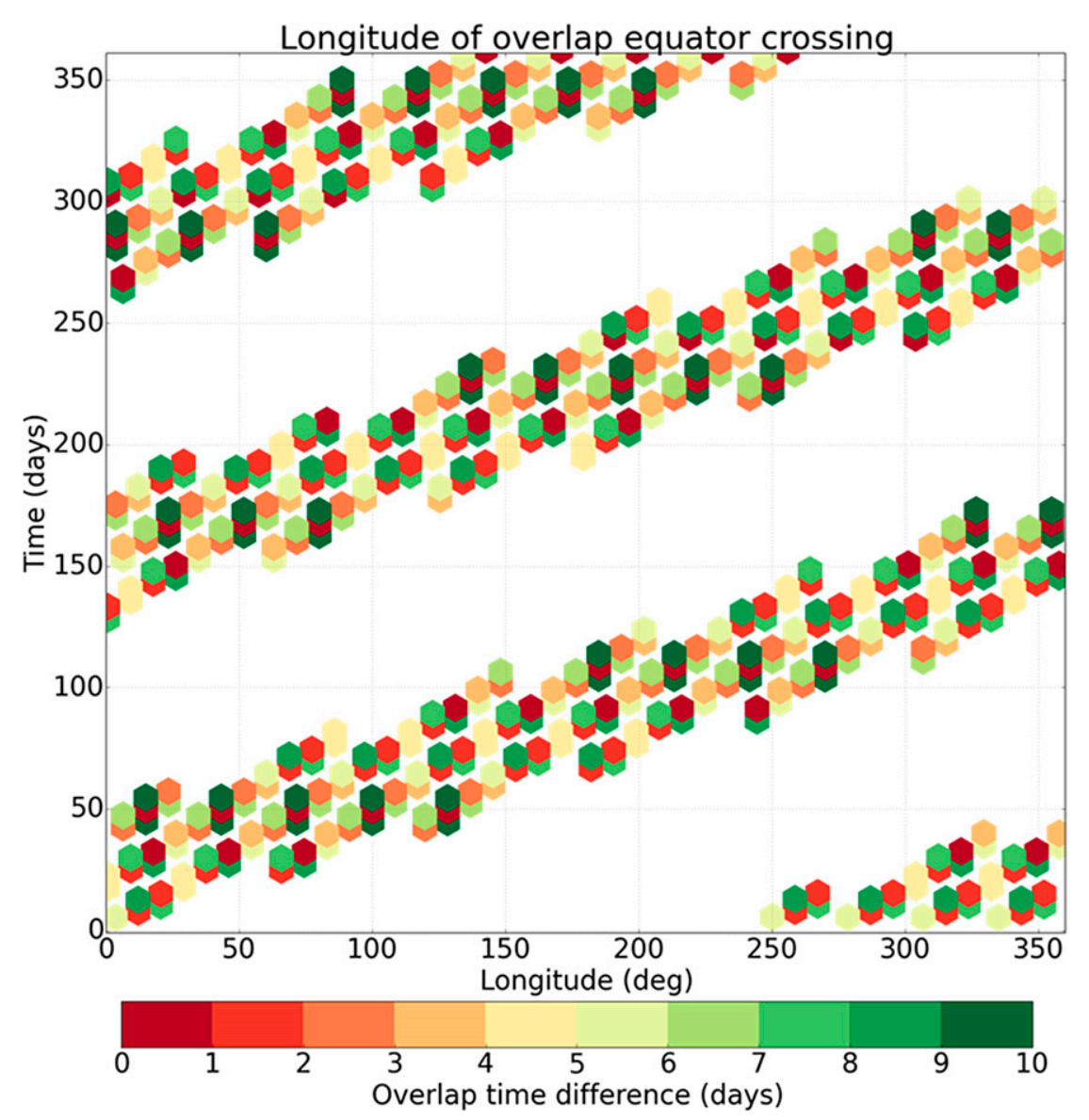

FIG. 8. As in Fig. 3b, but for overlaps between Jason-3 and an arbitrary Jason-2 EoL orbit $(12+286 / 399)$.

\section{b. Method used to look for Jason-2 EoL orbits}

These findings suggest we can maximize the value of a Jason-2 EoL geodetic orbit that meets the criteria used by Dibarboure et al. (2012) for good mesoscale and geodetic sampling, but also new criteria designed to provide good sampling of overlap events between Jason-2 EoL and Jason-3 (overlap sampling).

Maximizing the Jason-2 GM-Jason-3 overlap sampling means that overlap events should be homogeneously distributed in space (longitude of equator crossings) and in time (days throughout the EoL cycle) as opposed to the bad example in Fig. 8. Furthermore, for a given overlap time difference, the space-time distribution must also be as regular as possible (e.g., good distribution of 1-day overlaps).

We can gauge the homogeneity of the overlap sampling by splitting the space/time into bins (e.g., allocating each sample's equator crossing position and time into bins of $10^{\circ}$ and 30 days) and then counting how many overlap events occur in each space/time bin. We want to avoid empty bins, since the region would not be sampled for rapid overlapping events. Our analysis grid is based on the average number of overlaps per day between Jason-1 GM and Jason-2: the grid should be full and regularly sampled when overlap events are not aggregated. $^{2}$

We tested this methodology on the Jason-1 GM orbit. Figure 9 shows the distribution in longitude and time of overlap events (red dots) and the number of events in each bin (black and white background) for overlap events of 0-10 days (Fig. 9a) and 1 day (Fig. 9b). As expected from the previous discussions, Fig. 9a exhibits a rather homogeneous sampling, since only 12 bins are empty (out of 216 , i.e., $5 \%$ ). This should be compared to the bad orbit of Fig. 8, which has almost 50\% empty bins. Conversely,

\footnotetext{
${ }^{2}$ For overlaps of $0-10$ days, we use bins of $20^{\circ}$ and 30 days and the orbit is rejected if there are at least 15 empty bins. For overlaps of $0-1$ day, we use bins of $40^{\circ}$ and 60 days and the threshold is seven empty bins.
} 

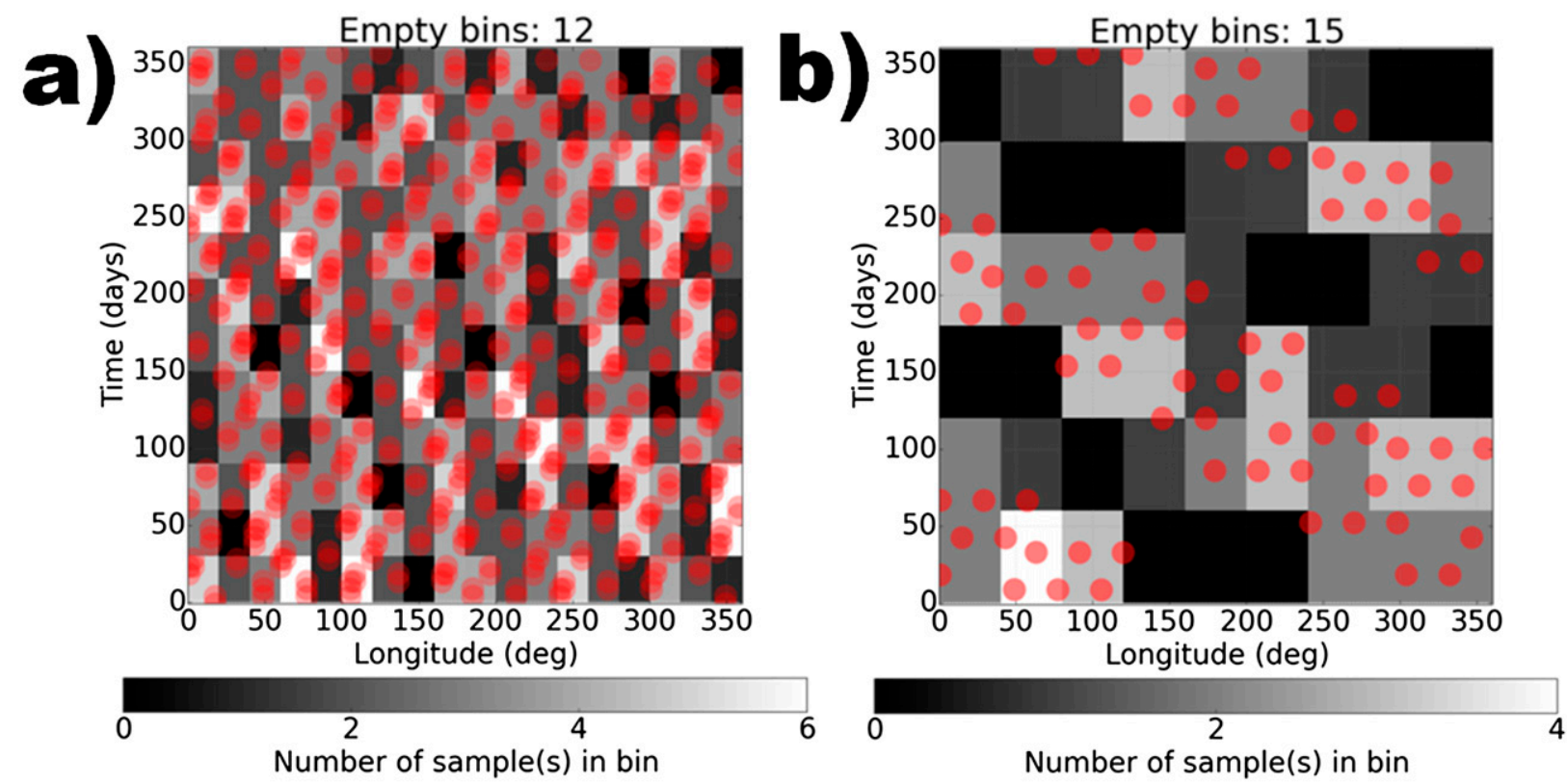

FIG. 9. (a) The longitude of the equator crossing of all tracks where Jason-1 GM is within $10 \mathrm{~km}$ and 10 days of Jason-2 (red dots). The background is the number of overlap events in $20^{\circ}$ per 30 -day bins (color scale). (b) As in (a), but for 1-day overlaps and $40^{\circ}$ per 60-day boxes.

Fig. 9b highlights the primary weakness of the Jason-1 GM orbit: the 0-1-day samples are aggregated in $100^{\circ} \times$ 100-day bands and there are no samples between these bands. As a result there are 15 empty bins (out of 36 , i.e., $27 \%$ ). Our Jason-2 screening filter would reject the Jason-1 GM orbit for this reason.

\section{c. Desirable orbit options for Jason-2 EoL}

We applied this screening methodology to various orbits recommended for the Jason-1 EoL by Dibarboure et al. (2012) and we found that all of them exhibited the same weakness as Jason-1 GM. So, we analyzed all of the EoL orbit options (more than 20000) and we slightly relaxed the geodetic and mesoscale criteria used for Jason-1 in order to keep more interesting orbits, even if they were not fully optimal on every criterion.

Our three screening filters (geodetic, mesoscale, and overlap) left 70 desirable options, generally located in small altitude bands (already reported by Dibarboure et al. 2012). For each altitude band, we manually selected only one candidate using the mesoscale sampling criteria.

The final list of orbits that are recommended for Jason-2 EoL is summarized in Fig. 10 (using the $N+P / Q$ terminology; i.e., the number of revolutions per nodal day), as well as their distribution in altitude and their strengths and weaknesses. In Fig. 10, column A gives the uniformity of the mesoscale sampling of Jason-2 EoL plus Jason-3 (derived from Dibarboure et al. 2012), column B gives the density and the uniformity of the geodetic sampling of Jason-2 EoL plus Jason-1 GM (derived from Dibarboure et al. 2012; also discussed in section 3d), column $\mathrm{C}$ is the overlap criteria from section $2 b$, and column D gives the collision risk due to debris at each altitude (courtesy of the Jason-2 operations team). The red box in the left panel shows the forbidden altitude zone, where there is a high risk of collision with the now uncontrolled TOPEX and Jason-1 platforms.

The best option in this list is arguably $12+284 / 371$ at $1309 \mathrm{~km}(27 \mathrm{~km}$ lower than the nominal TOPEX altitude) for the following reasons (1-3 are the criteria used for Jason-1 GM):

1) It has a 17-day subcycle that is good for mesoscale monitoring because it blends well with the 10-day cycle of Jason-3.

2) It has a 145-day subcycle and a 371-day repeat cycle that are good for geodesy: the final grid is close to the Jason-1 GM grid. If Jason-2 EoL was to die after only half the repeat cycle, it would still provide a coarser but globally homogeneous dataset for geodetic users.

3) It has a 4-day subcycle that is favorable for sea-state applications (e.g., assimilation in operational wave models) and that blends well with Jason-3's 3-day subcycle.

4) It generates overlap events with Jason-3 that are well distributed at all time scales. There are no empty bins for the 10-day criterion, and only three empty bins for the 1-day criterion (not shown). This orbit yields a 


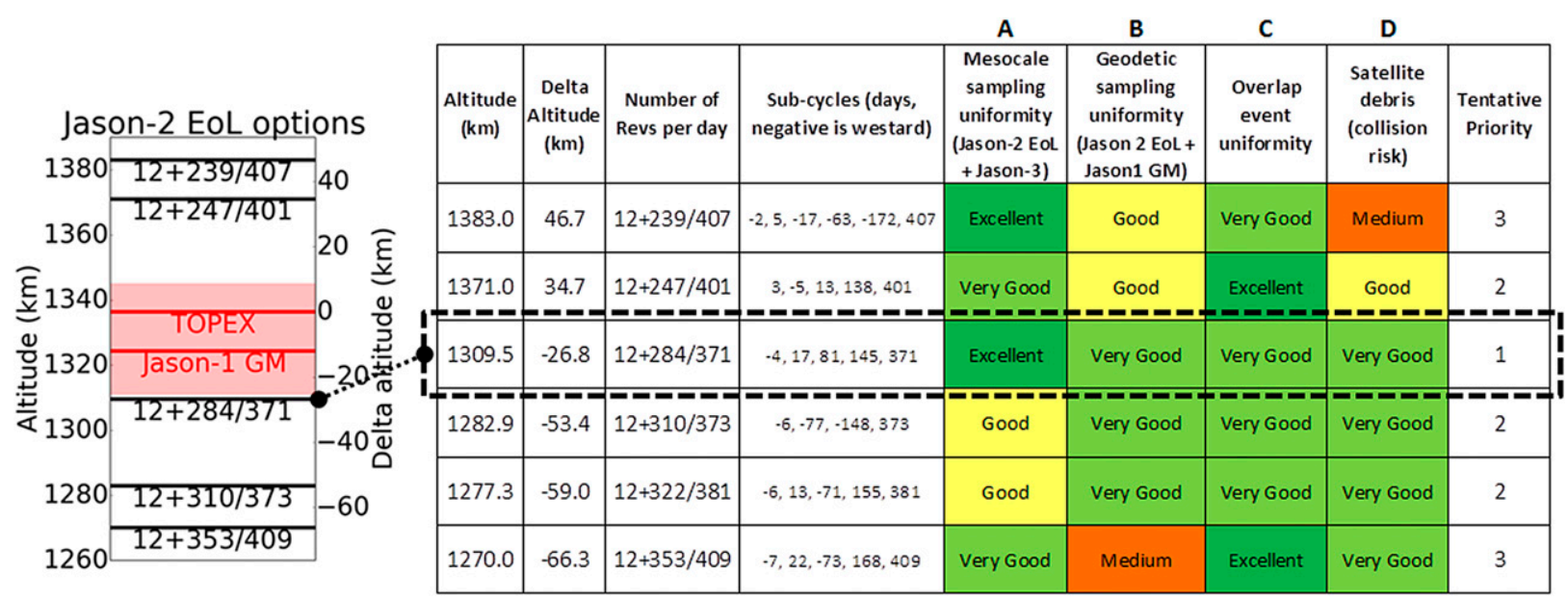

FIG. 10. Shortlist of desirable options for Jason-2 EoL: priority list with subcycles and altitude distribution (absolute or relative to the TOPEX/Poseidon historical orbit) and qualitative value with respect to selection criteria of Dibarboure et al. (2012) and section 3b.

high probability of collecting an overlap sample in any region, season, and for any time difference.

5) It is far from the uncontrolled TOPEX and Jason-1 platforms and from the debris orbiting near $1400 \mathrm{~km}$ (e.g., from the Globalstar constellation).

Figure 11 shows some characteristics of the $12+284 / 371$ orbit. Figure 11a shows the equator-crossing longitude for each ascending pass (red is Jason-3 on the TOPEXJason orbit, blue is the proposed Jason-2 EoL). The black circles highlight what happens when there is an overlap with an almost 0-day time difference. Jason-3 and Jason-2 EoL are measuring the same topography for the pass with the black dashed circle. In this configuration nearby Jason-2 EoL tracks (dotted circles) are interleaved with Jason-3, thus reducing local duplication and mesoscale sampling loss (mesoscale criterion used by Dibarboure et al. 2012). Figure 11b gives the longitude of equator crossings for all overlap events with Jason-3. This panel essentially synthesizes why this orbit passed the screening filters of section $3 \mathrm{~b}$.

\section{d. Geodetic sampling}

Jason-1 GM has collected a valuable dataset (Sandwell et al. 2014) with a resolution on the order of $8 \mathrm{~km}$, so it would be natural to use Jason-2 EoL to improve the geodetic record resolution. In theory, this would be achieved by putting Jason-2 EoL exactly between Jason-1 GM tracks to minimize the duplication between both altimeters and to achieve a resolution of $4 \mathrm{~km}$. Unfortunately, an interleaved geodetic mission would require Jason-2 EoL to use exactly the altitude of Jason-1 GM, but this is impossible due to collision risks between both satellites.
Any other geodetic orbit will provide irregular sampling between the Jason-1 GM tracks: in certain regions, Jason-2 EoL will be perfectly interleaved with the Jason-1 GM geodetic grid, and in others they will be almost exactly on the same grid. These so-called moiré patterns appear when two grids of different resolution are superimposed.

Figure $11 \mathrm{c}$ shows the longitude of ascending equator crossings for one full geodetic cycle of Jason-1 GM (blue) and Jason-2 EoL (red). The moiré bands are the alternating lighter/darker zones where the red and blue lines meet: in certain regions Jason-2 EoL would duplicate measurements from Jason-1 GM (resolution on the order of $8 \mathrm{~km}$ ), whereas in others Jason-2 EoL would complement the Jason-1 GM grid (resolution of $4 \mathrm{~km}$ ).

To illustrate the impact of the moiré patterns, the black line of Fig. 11c uses a maximum kernel density estimator: this $4-\mathrm{km}$ radius estimator will be locally maximal when the two grids are perfectly interleaved and minimal when they are perfectly overlapping. The density estimator increases near altimeter tracks but when tracks are too close, they essentially provide duplicate measurements and the observation quality does not increase, resulting in 8 -km oscillations modulated by larger arches (moiré bands) that are $41 \mathrm{~km}$ long.

The global geodetic sampling capability is similar for all of the selected EoL orbits. In contrast, there are differences in the size of the moiré patterns: the moiré patterns of the orbits listed in Fig. 10 were measured and they range from 20 to $500 \mathrm{~km}$ (not shown). Shorter moiré bands $(20-40 \mathrm{~km})$ as in Fig. 11c are slightly more desirable, as they spread out duplication and blind zones instead of aggregating them in large unobserved bands. 

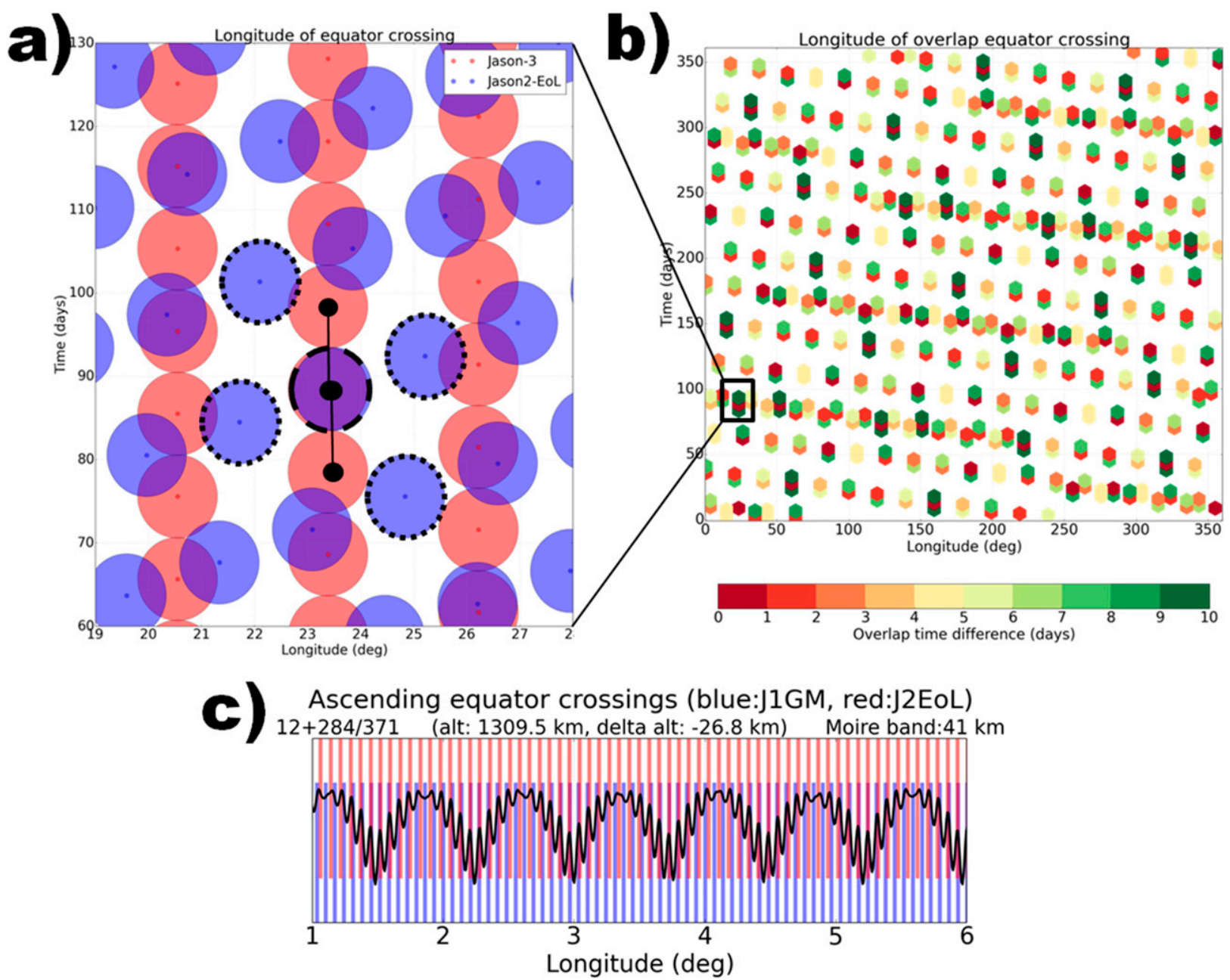

FIG. 11. As in Fig. 3, but for overlaps between Jason-3 and a tentative Jason-2 EoL orbit (namely, $12+284 / 371$ at 1309 km). (a) The black circles in highlight the mesoscale research criteria used by Dibarboure et al. (2012). (c) The moiré pattern (41 km bands at the equator) between the geodetic tracks of Jason-1 GM (blue) and Jason-2 EoL (red).

This is accounted for in the column B diagnostics in Fig. 10.

\section{Summary and conclusions}

Because of the drifting nature of the orbit used for Jason-1 during its geodetic phase, and the geometry of its ground track (almost the same as for the Jason-2 orbit), there are 1200 overlap events where both altimeters are measuring the ocean topography over the same track (less than $10 \mathrm{~km}$; i.e., the altimeter footprint radius) over an entire pass, that is, thousands of kilometers. The overlap events sample homogeneously all longitudes and all time differences.

When the time difference is almost zero, the difference is characterized by $1-\mathrm{Hz}$ altimeter noise and its modulation by waves. As $d t$ increases, the rapid ocean variability is revealed. Our statistical analysis yields variance maps, spectra, autocorrelation, and space-time scales that are consistent with past observations (e.g., 3-day phase of $E R S-1)$. These first findings highlight the value of this Jason-1 GM overlap dataset for more sophisticated studies of the rapid ocean variability but also two major limitations: 1) the noise level of Jason-class altimeters prevents us from analyzing scales smaller than $80 \mathrm{~km}$ and 2) short time differences also absorb a fraction of the derivative of slower signals.

These findings are important for Jason-2 if a geodetic phase is considered in the coming years (e.g., when the satellite starts to exhibit aging problems) because Jason-2 GM and Jason-3 have the potential to collect a better dataset if Jason-2 GM uses an orbit with specific properties. By extending the search criteria used by Dibarboure et al. (2012) for Jason-1 EoL with new criteria 

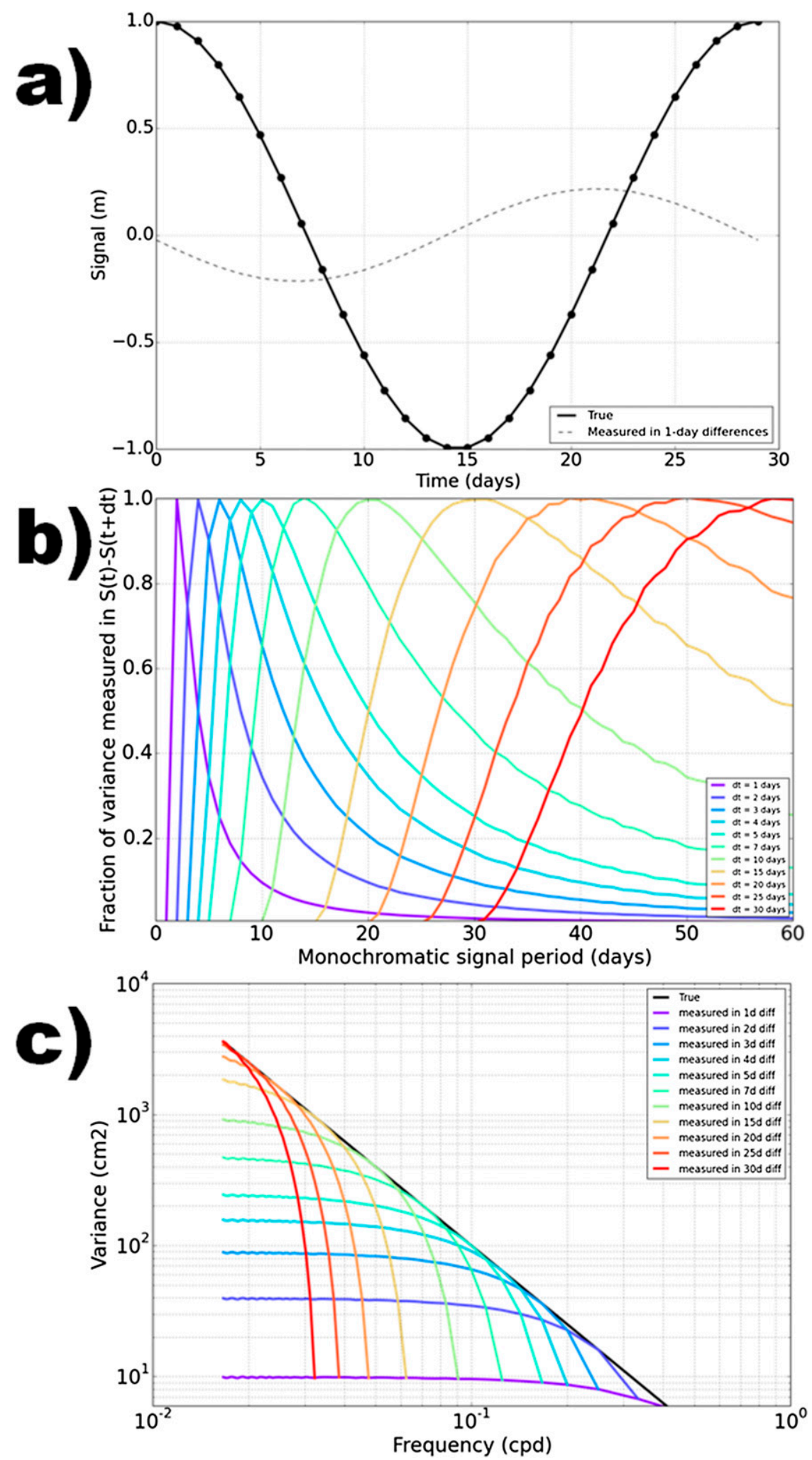

FIG. A1. Frequency sampling in time differences $d t$. (a) A simple monochromatic signal with a period of 30 days (plain) and what is measured in 1-day differences of subsequent points (dashed). (b) The fraction of variance of a monochromatic signal measured in $S(t+d t)-S(t)$ as a function of the signal period and $d t$. (c) The PSD of 1- to 30-day differences (colors) if the true signal PSD is following an $f^{2}$ power law (black line). 
designed to guarantee that overlap events are homogeneously distributed in space and time, we selected a small subset of desirable orbits.

The subset of collocated Jason-1 and Jason-2 tracks used in this study can be made available to other scientific users for further studies on the altimeter noise and its geographical modulation, or on the rapid ocean dynamics. The selection of tracks is provided as supplementary material (refer to JTECH-D-16-0015.s1).

Acknowledgments. This work was supported by CNES (SALP and SWOT projects; Contract 104685/00). It was stimulated by discussions from the Ocean Surface Topography Science Team tasked with evaluating the scientific merits of a Jason-2 EoL phase, and from the SWOT Science Definition Team (goals of the exact 1-day repeat orbit of SWOT). We thank Drs. P-Y. Le Traon and C. Ubelmann for their insightful comments and suggestions, and the three anonymous reviewers for their thorough and helpful comments on the original manuscript.

\section{APPENDIX}

\section{What is Measured in Jason-1 GM-Jason-2 differences?}

Because of the nonrepeat nature of overlap events between Jason-1 GM and Jason-2, the observations are randomly distributed samples with 0- to 60-day differences. The major caveat is that a 1-day difference is measuring a wide range of frequencies, not just 1-day variability. Figure A1 illustrates this effect with simple monochromatic signals having periods ranging from 2 to 60 days.

Figure A1(a) shows an example for a 30-day signal (plain line) that is partially observed through 1-day differences (i.e., difference between subsequent dotted points). If the time difference is small with respect to the true period (here, 1 day vs 30 days), then the difference includes a derivative of the true signal and captures a fraction of its variance. Repeating this process for all periods and all time differences, we obtain Fig. A1(b), which shows the fraction of the true signal variance that is absorbed in a SLA difference for $d t$ ranging from 1 to 30 days. To illustrate, the 3-day differences absorb the variance of a wide range of monochromatic signals with periods ranging from 1.5 days ( $d t / 2$ - aliasing) to 30 days with a peak for monochromatic signals with a period of 6 days $(2 d t)$. The folding of long periods into the Jason-2-Jason-1 differences decreases with the period $\omega$ following a power law of $\omega^{-2}$, that is, $f^{+2}$, where $f$ is the frequency.

Assuming that the ocean PSD or variance is following an $f^{-2}$ law (as per Wortham and Wunsch 2014), we obtain
Fig. A1(c), which yields an approximation of the frequencies contained in 1- to 30-day differences. For a time difference of $d t$, the spectrum is flat for periods longer than $2 d t$. The spectra then decrease slowly for periods close to $d t$, and then decrease rapidly for periods smaller than $d t / 2$. Thus, measurements based on time difference $d t$ are essentially merging equally all periods that are substantially longer than $d t$ (observation of the derivative) and as small as $d t / 2$ (aliasing). Figure A1 is therefore interesting as a warning that Jason-1 GM-Jason-2 differences should be interpreted with care, since slower variability is assuredly affecting our results.

\section{REFERENCES}

Amarouche, L., L. Zawadzki, A. Vernier, G. Dibarboure, S. Labroue, M. Raynal, and J. Poisson, 2014: Reduction of the sea surface height spectral hump using a new retracker decorrelating ocean estimated parameters (DCORE). Preprints, 2014 Ocean Surface Topography Science Team Meeting, Konstanz, Germany, AVISO. [Available online at http://meetings.aviso.altimetry.fr/fileadmin/ user_upload/tx_ausyclsseminar/files/28Ball1615-3_DCORE_ OSTST2014_v5.pdf.]

AVISO, 2015: SSALTO/DUACS user handbook: (M)SLA and (M)ADT near-real time and delayed time products. CLSDOS-NT-06-034, SALP-MU-P-EA-21065-CLS, CNES, 66 pp. [Available online at http://www.aviso.oceanobs.com/fileadmin/ documents/data/tools/hdbk_duacs.pdf.]

Chelton, D. B., J. C. Ries, B. J. Haines, L. L. Fu, and P. S. Callahan, 2000: Satellite altimetry. Satellite Altimetry and Earth Sciences: A Handbook of Techniques and Applications, L.-L. Fu and A. Cazenave, Eds., International Geophysics Series, Vol. 69, Academic Press, 1-131.

Dibarboure, G., and J. Lambin, 2015: Monitoring the ocean surface topography virtual constellation: Lessons learned from the contribution of SARAL/AltiKa. Mar. Geod., 38, 684-703, doi:10.1080/01490419.2014.984883.

—, M.-I. Pujol, F. Briol, P.-Y. Le Traon, G. Larnicol, N. Picot, F. Mertz, and M. Ablain, 2011: Jason-2 in DUACS: First tandem results and impact on processing and products. Mar. Geod., 34, doi:10.1080/01490419.2011.584826.

_ and Coauthors, 2012: Finding desirable orbit options for the "Extension of Life"” phase of Jason-1. Mar. Geod., 35 (Suppl.), 363-399, doi:10.1080/01490419.2012.717854.

_, F. Boy, J.-D. Desjonquères, S. Labroue, Y. Lasne, N. Picot, J.-C. Poisson, and P. Thibaut, 2014: Investigating short-wavelength correlated errors on low-resolution mode altimetry. J. Atmos. Oceanic Technol., 31, 1337-1362, doi:10.1175/JTECH-D-13-00081.1.

Dorandeu, J., M. Ablain, and P.-Y. Le Traon, 2003: Reducing cross-track geoid gradient errors around TOPEX/Poseidon and Jason-1 nominal tracks: Application to calculation of sea level anomalies. J. Atmos. Oceanic Technol., 20, 1826-1838, doi:10.1175/1520-0426(2003)020<1826:RCGGEA > 2.0.CO;2.

Ducet, N., P.-Y. Le Traon, and G. Reverdin, 2000: Global high resolution mapping of ocean circulation from the combination of TOPEX/POSEIDON and ERS-1/2. J. Geophys. Res., 105, 19 477-19498, doi:10.1029/2000JC900063.

Dufau, C., M. Orsztynowicz, G. Dibarboure, R. Morrow, and P.-Y. Le Traon, 2016: Mesoscale resolution capability of altimetry: Present and future. J. Geophys. Res. Oceans, 121, 4910-4927, doi:10.1002/2015JC010904. 
Farrar, J. T., and T. S. Durland, 2012: Wavenumber-frequency spectra of inertia-gravity and mixed Rossby-gravity waves in the equatorial Pacific Ocean. J. Phys. Oceanogr., 42, 18591881, doi:10.1175/JPO-D-11-0235.1.

Garcia, E., D. Sandwell, and W. Smith, 2014: Retracking CryoSat-2, Envisat and Jason-1 radar altimetry waveforms for improved gravity field recovery. Geophys. J. Int., 196, 1402-1422, doi:10.1093/gji/ggt469.

Le Traon, P.-Y., 1991: Time scales of mesoscale variability and their relationship with space scales in the North Atlantic. J. Mar. Res., 49, 467-492, doi:10.1357/002224091784995828.

—_, and F. Ogor, 1998: ERS-1/2 orbit improvement using TOPEX/POSEIDON: The $2 \mathrm{~cm}$ challenge. J. Geophys. Res., 103, 8045-8057, doi:10.1029/97JC01917.

— M.-C. Rouquet, and C. Boissier, 1990: Spatial scales of mesoscale variability in the North Atlantic as deduced from Geosat data. J. Geophys. Res., 95, 20 267-20 285, doi:10.1029/ JC095iC11p20267.

, and Coauthors, 2015: Use of satellite observations for operational oceanography: Recent achievements and future prospects. J. Oper. Oceanogr., 8 (Suppl.), s12-s27, doi:10.1080/ 1755876X.2015.1022050.

Minster, J.-F., and M.-C. Gennero, 1995: High-frequency variability of western boundary currents using ERS 1 three-day repeat altimeter data. J. Geophys. Res., 100, 22 603-22 612, doi:10.1029/94JC02401.

Samelson, R., M. Schlax, and D. Chelton, 2014: Randomness, symmetry and scaling of mesoscale eddy life cycles. J. Phys. Oceanogr., 44, 1012-1029, doi:10.1175/JPO-D-13-0161.1.

Sandwell, D., R. Dietmar Müller, W. Smith, E. Garcia, and R. Francis, 2014: New global marine gravity model from CryoSat-2 and Jason-1 reveals buried tectonic structure. Science, 346, 65-67, doi:10.1126/science.1258213.

Stammer, D., 1997: Global characteristics of ocean variability estimated from regional TOPEX/POSEIDON altimeter measurements. J. Phys. Oceanogr., 27, 1743-1769, doi:10.1175/ 1520-0485(1997)027<1743:GCOOVE > 2.0.CO;2.

Wortham, C., and C. Wunsch, 2014: A multidimensional spectral description of ocean variability. J. Phys. Oceanogr., 44, 944966, doi:10.1175/JPO-D-13-0113.1. 